\title{
Mortes infantis evitáveis por intervenções do Sistema Único de Saúde: comparação de duas coortes de nascimentos
}

\author{
Avoidable causes of infant deaths due to interventions of the \\ Brazilian Unified Health System: a comparison of two birth cohorts
}

Hellen Geremias dos Santos ${ }^{1}$

Selma Maffei de Andrade ${ }^{1}$

Ana Maria Rigo Silva ${ }^{1}$

Thais Aidar de Freitas Mathias ${ }^{2}$

Lígia Lopes Ferrari ${ }^{3}$

Arthur Eumann Mesas ${ }^{1}$

\footnotetext{
${ }^{1}$ Departamento de Saúde Coletiva, Universidade Estadual de Londrina. Av. Robert Koch 60, Vila Operária. 86.038-350 Londrina PR Brasil. hellengeremias@gmail.com ${ }^{2}$ Departamento de Enfermagem, Centro de Ciências da Saúde, Universidade Estadual de Maringá.

${ }^{3}$ Departamento de Pediatria e Cirurgia Pediátrica, Centro de Ciências da Saúde, Universidade Estadual de Londrina.
}

Abstract Avoidable infant mortality was compared in the cohorts of live births in 2000/20001 and 2007/2008 in Londrina in the state of Paraná. Data on live births and infant deaths were matched in a single database and the underlying causes of death were classified according to the Brazilian List of Avoidable Causes of Mortality of the Brazilian Unified Health System. The proportional variations of death rates were calculated and the technique of correspondence analysis was used. The proportions of avoidable deaths were $71.6 \%$ in 2000/2001 and 65.5\% in 2007/2008. Avoidable mortality rate due to "care of the woman during pregnancy" was the most frequent in the two biennials with an increase of $16.7 \%$. There was a decline in avoidable mortality rates due to other causes. In 2000/2001, correspondence analysis revealed a profile of deaths characterized mainly by causes avoidable by "care of the woman during labor" and "health promotion/care" while, in 2007/2008, by causes avoidable by "care of the woman during pregnancy," particularly among low birth weight newborns and "not clearly avoidable," among those with adequate weight. Despite a reduction in some avoidable causes of deaths, improvements in prenatal care are required.

Key words Infant mortality, Causes of death, Health service evaluation
Resumo Comparou-se a mortalidade infantil evitável nas coortes de nascimentos de 2000/2001 e de 2007/2008, em Londrina-PR. Dados sobre nascidos vivos e óbitos infantis foram vinculados em base de dados única e as causas básicas de morte foram agrupadas conforme a Lista Brasileira de Causas de Mortes Evitáveis por Intervenções do Sistema Único de Saúde. Calculou-se a variação percentual das taxas de mortes entre os biênios e usou-se a técnica de análise de correspondência. As proporções de óbitos evitáveis foram de 71,6\% em 2000/2001 e de 65,5\% em 2007/2008. A taxa de mortalidade evitável por atenção à mulher na gestação predominou nos dois biênios, com incremento de $16,7 \%$. Houve declínio das taxas de mortalidade evitável pelas demais causas. A análise de correspondência mostrou, em 2000/2001, um perfil de mortes representado principalmente por causas evitáveis por atenção à mulher no parto e por adequada promoção/atenção à saúde, enquanto que, em 2007/2008, por causas evitáveis por adequada atenção à mulher na gestação, especialmente entre os nascidos com baixo peso, e não claramente evitáveis entre os com peso adequado. Apesar da diminuição da mortalidade por algumas causas evitáveis, são necessárias medidas para aumentar a qualificação da atenção durante a gestação.

Palavras-chave Mortalidade infantil, Causas de morte, Avaliação de serviços de saúde 
Introdução

A taxa de mortalidade infantil (TMI), além de ser um bom indicador das condições de saúde, expressa o desenvolvimento econômico e a qualidade de vida de uma população. No Brasil, entre 1990 e 2007, esse indicador apresentou declínio em todas as regiões brasileiras, especialmente nas menos desenvolvidas ${ }^{1,2}$, e as melhorias socioeconômicas e de acesso aos serviços de saúde foram as principais responsáveis por essas reduções. Entre essas melhorias, destacam-se diminuições da desigualdade de renda, aumento da escolaridade e da inserção da mulher no mercado de trabalho, e expansão da Estratégia Saúde da Família (ESF) e de tecnologias em assistência neonatal ${ }^{2,3}$. No entanto, apesar desse progresso, atualmente mais da metade das mortes infantis ocorridas no País é considerada evitável ${ }^{4}$.

A atribuição de evitabilidade aos óbitos infantis tem ganhado atenção especial em anos recentes por potencializar o reconhecimento tanto de ações exitosas como de lacunas ainda existentes no processo de trabalho e na organização dos serviços de saúde voltados à atenção de mulheres e crianças ${ }^{5}$. Nesse sentido, foi desenvolvida a Lista Brasileira de Causas de Mortes Evitáveis por Intervenções do Sistema Único de Saúde (SUS) com base nas causas de morte que poderiam ser prevenidas, ou diagnosticadas e tratadas precocemente, por meio de tecnologia ofertada pelo SUS ${ }^{5,6}$.

Bases de dados dos Sistemas de Informações sobre Nascidos Vivos (SINASC) e sobre Mortalidade (SIM) e registros de comitês de mortalidade infantil configuram fontes de dados dos serviços de saúde para o monitoramento de causas de morte infantil. Em Londrina, Paraná, tem se registrado boa qualidade da informação sobre nascidos vivos ${ }^{7}$ e há um comitê de prevenção da mortalidade infantil atuante desde 1999, o que também tem possibilitado informações mais fidedignas sobre causas de óbitos infantis. Assim, considerando a existência de uma lista brasileira de causas de mortes evitáveis por intervenções do SUS, já utilizada em outros estudos para identificação dessas causas ${ }^{4,8}$, a boa qualidade de dados sobre eventos vitais em Londrina, e a importância de avaliações de possíveis mudanças nos perfis de mortes infantis evitáveis para o planejamento de ações de saúde, este estudo objetivou comparar a mortalidade infantil evitável em duas coortes de nascidos vivos, nos biênios 2000/2001 e 2007/2008, por meio da Lista Brasileira de Causas de Mortes Evitáveis por Intervenções do Sistema Único de Saúde $e^{5,6}$.

\section{Métodos}

O estudo foi realizado em Londrina, Paraná, município com população de 506.701 habitantes em $2010^{9}$. Trata-se de um estudo exploratório e comparativo da evitabilidade de óbitos infantis das coortes de nascidos vivos (NV) de dois biênios nos anos 2000. O biênio 2000/2001 foi selecionado por ser subsequente à implantação do Comitê Municipal de Prevenção da Mortalidade Materna e Infantil (CMPMMI) em Londrina, e por anteceder a expansão das equipes da ESF no Município. A segunda coorte referiu-se ao biênio 2007/2008, por ser o mais recente com dados sobre nascidos vivos à época da coleta de dados deste estudo.

Para a formação das coortes, os bancos de dados do SINASC de 2000/2001 e de 2007/2008 foram obtidos no sítio eletrônico do Datasus ${ }^{10}$. Foram selecionados os registros de NV de mães residentes em Londrina com ocorrência do parto no Estado do Paraná, os quais representam mais de $99 \%$ dos nascimentos de residentes no Município. Os óbitos infantis foram identificados no banco de dados do $\operatorname{SIM}^{10}$ e nas fichas de investigação preenchidas pelo CMPMMI de 2000 a 2002 e de 2007 a 2009. Os registros do CMPMMI foram consultados por não se ter certeza sobre a devida correção da causa básica de morte no SIM após investigação do processo que culminou na morte infantil. Esses registros possibilitaram, ainda, a obtenção do número da declaração de nascido vivo (DN) para 2000/2001, campo não disponível no SIM naquele período.

Os óbitos foram relacionados ao banco de dados de NV por linkage determinístico, usando o número da DN como informação-chave. Em caso de ausência desse dado no CMPMMI/SIM, as características maternas e do recém-nascido foram utilizadas para pareamento ${ }^{11}$.

A causa básica de morte dos óbitos pareados às coortes de nascimento, atribuída pelo CMPMMI após investigação, ou identificada no banco de dados do SIM para os óbitos não investigados, foi codificada segundo a Classificação Estatística Internacional de Doenças e Problemas Relacionados à Saúde, Décima Revisão (CID-10). Posteriormente, os códigos das causas básicas de morte foram agrupados conforme a Lista Brasileira de Causas de Mortes Evitáveis por Intervenções do Sistema Único de Saúde ${ }^{5,6}$, em:

. Causas evitáveis: (a) reduzíveis por ações de imunoprevenção; (b) reduzíveis por adequada atenção à mulher na gestação e parto e ao feto e ao recém-nascido; (c) reduzíveis por ações adequa- 
das de diagnóstico e tratamento; (d) reduzíveis por ações adequadas de promoção à saúde, vinculadas às ações adequadas de atenção à saúde;

- Causas mal definidas;

. Demais causas, não claramente evitáveis.

Para comparação da mortalidade infantil evitável entre 2000/2001 e 2007/2008, foram calculados os seguintes indicadores: taxas de mortalidade infantil, neonatal (precoce e tardia), pósneonatal e por causas evitáveis, além da variação percentual (VP) das taxas de mortalidade por causas. Adicionalmente, utilizou-se análise de correspondência, feita com o programa Statistica versão 10.0, para identificação de modificações no perfil de óbitos entre os biênios. Este método permite a visualização de relações entre categorias de variáveis por meio da distância entre pontos desenhados em gráficos. Para a análise, utilizou-se uma matriz com dados referentes às taxas de mortalidade segundo cada grupo de causas (colunas), para os respectivos biênios (linhas). Também foi apresentada a inércia relativa das categorias de cada variável. Esse dado referese ao papel que cada categoria exerce na construção do eixo (dimensão) do gráfico, indicando categorias altamente correlacionadas com o eixo e entre $\mathrm{si}^{12}$.

As causas específicas dos subgrupos de causas evitáveis foram analisadas conforme classificação da CID-10. A evitabilidade dos óbitos infantis também foi analisada por faixas de peso ao nascer: $<2500 \mathrm{~g}$ (baixo peso) e $\geq 2500 \mathrm{~g}$ (peso adequado).

O estudo foi aprovado pelo Comitê de Ética em Pesquisa da Universidade Estadual de Londrina e seguiu as diretrizes da Resolução 196/96 13 do Conselho Nacional de Saúde.

\section{Resultados}

Em 2000/2001 foram identificados $15.385 \mathrm{NV}$ e 180 óbitos infantis dessa coorte, dos quais apenas um não investigado pelo CMPMMI e, em 2007/2008, 13.208 NV e 148 óbitos infantis, todos investigados.

As taxas de mortalidade infantil foram de 11,70 e 11,20 por $1000 \mathrm{NV}$ em 2000/2001 e em 2007/2008, respectivamente. Houve predomínio da mortalidade neonatal nos dois biênios, sobretudo da neonatal precoce, com taxas de 6,24 e 6,36 óbitos por 1000 NV, em 2000/2001 e 2007/ 2008, respectivamente. A taxa de mortalidade neonatal tardia apresentou o maior aumento entre os períodos estudados: de 1,82 óbitos a cada
1000 NV, no primeiro biênio, para 2,27 no biênio recente. A taxa de mortalidade pós-neonatal foi a única que apresentou redução, passando de 3,64 óbitos a cada 1000 NV, em 2000/2001, para 2,57 em 2007/2008.

Quanto à evitabilidade, 71,6\% dos óbitos da coorte de 2000/2001 e 65,5\% dos da coorte de $2007 / 2008$ foram considerados evitáveis por intervenções do SUS, com taxas de, respectivamente, 8,38 e 7,34 óbitos por 1000 NV. Os óbitos evitáveis por adequada atenção à mulher durante a gestação e o parto e ao feto e ao recém-nascido foram predominantes (taxas de 6,82 e 6,51 óbitos por $1000 \mathrm{NV}$, respectivamente, em 2000/2001 e 2007/2008), com destaque para o subgrupo adequada atenção à mulher na gestação nos dois biênios (Tabela 1). Quando os subgrupos foram analisados separadamente, observou-se declínio de $22,2 \%$ na taxa de mortalidade evitável por adequada atenção à mulher no parto, devido especialmente à redução de óbitos cujas causas básicas foram "outras complicações do trabalho de parto ou do parto que afetam o recém-nascido" (código da CID-10: P03). A mortalidade evitável por adequada atenção ao feto e ao recémnascido diminuiu em $64,6 \%$, principalmente pela queda do número de óbitos por infecções específicas do período perinatal (códigos P35-P39), pneumonias congênitas não especificadas (código P23.9) e por outros transtornos originados no período perinatal (códigos P90-P96). Por outro lado, a taxa de mortalidade evitável por adequada atenção à mulher na gestação apresentou aumento de $16,7 \%$, devido ao incremento de mortes infantis por transtornos maternos hipertensivos (código P00.0) e por doenças maternas renais e das vias urinárias (P00.1).

Houve redução de seis para um óbito evitável por ações adequadas de diagnóstico e tratamento (Tabela 1), principalmente pela diminuição de mortes por infecções do aparelho respiratório (códigos J06.9 e J21.9) e por outras infecções bacterianas (códigos A39 e A41.9). As mortes evitáveis por ações adequadas de promoção à saúde, vinculadas às ações adequadas de atenção à saúde reduziram-se de 17 para 10 e este declínio ocorreu devido, especialmente, às inalações do conteúdo gástrico (código W78).

As causas não claramente evitáveis apresentaram aumento de 24,3\% (Tabela 1). A maior frequência de malformações congênitas como causa básica de morte dos óbitos da coorte de 2007/ 2008 foi fator determinante desse incremento. Entre as mortes por malformações congênitas, as não especificadas foram as mais frequentes no 
primeiro biênio e, no segundo, destacaram-se as do aparelho circulatório (códigos Q20-Q28). A causa básica de morte foi classificada como mal definida em quatro óbitos de NV em 2000/2001, e em apenas um de 2007/2008 (Tabela 1).

Entre os recém-nascidos do primeiro biênio que morreram, 43,9\% apresentaram peso ao nascer < 1500g, 24,4\% peso entre 1500 e $2499 \mathrm{~g}$ e $31,7 \%$ peso $\geq 2500 \mathrm{~g}$. No biênio mais recente, os óbitos de NV com peso < 1500g corresponderam a 48,6\%, os com peso entre 1500 e $2499 \mathrm{~g}$ a $18,2 \%$ e os com peso adequado a $33,1 \%$.

A taxa de mortalidade infantil evitável entre NV com baixo peso reduziu-se em 5,1\% (Tabela 2). Em ambos os biênios, os óbitos desse grupo poderiam ser evitados mais frequentemente por adequada atenção à mulher na gestação, com incremento de $18,5 \%$ na taxa específica desse

Tabela 1. Óbitos infantis (no, taxa por mil nascidos vivos e variação percentual), segundo a Lista Brasileira de Mortes Evitáveis, 2000/2001 e 2007/2008, Londrina (PR).

\begin{tabular}{|c|c|c|c|c|c|}
\hline \multirow[b]{2}{*}{ Grupo de causas } & \multicolumn{2}{|c|}{$2000 / 2001$} & \multicolumn{2}{|c|}{$2007 / 2008$} & \multirow[b]{2}{*}{ VP } \\
\hline & Óbitos & TMI & Óbitos & TMI & \\
\hline Causas de morte evitáveis & 129 & 8,38 & 97 & 7,34 & $-12,4$ \\
\hline Reduzíveis por ações de imunoprevenção & 1 & 0,06 & - & - & - \\
\hline $\begin{array}{l}\text { Reduzíveis por adequada atenção à mulher na } \\
\text { gestação e parto e ao feto e ao recém-nascido }\end{array}$ & 105 & 6,82 & 86 & 6,51 & $-4,5$ \\
\hline atenção à mulher na gestação & 59 & 3,83 & 59 & 4,47 & $+16,7$ \\
\hline atenção à mulher no parto & 36 & 2,34 & 24 & 1,82 & $-22,2$ \\
\hline atenção ao feto e ao recém nascido & 10 & 0,65 & 3 & 0,23 & $-64,6$ \\
\hline $\begin{array}{l}\text { Reduzíveis por ações adequadas de diagnóstico e } \\
\text { tratamento }\end{array}$ & 6 & 0,39 & 1 & 0,08 & $-79,5$ \\
\hline $\begin{array}{l}\text { Reduzíveis por ações adequadas de promoção à saúde, } \\
\text { vinculadas a ações adequadas de atenção à saúde }\end{array}$ & 17 & 1,10 & 10 & 0,76 & $-30,9$ \\
\hline Causas de morte mal-definidas & 4 & 0,26 & 1 & 0,08 & $-69,2$ \\
\hline Demais causas (não claramente evitáveis) & 47 & 3,05 & 50 & 3,79 & $+24,3$ \\
\hline Total & 180 & 11,70 & 148 & 11,20 & $-4,2$ \\
\hline
\end{tabular}

TMI: taxa de mortalidade infantil; VP: variação percentual das taxas.

Tabela 2. Óbitos infantis ( $\mathrm{n}^{\circ}$, taxa por mil nascidos vivos e variação percentual) de crianças nascidas com baixo peso $(<2500 \mathrm{~g})$ e com peso adequado ( $\geq 2500 \mathrm{~g})$, segundo a Lista Brasileira de Mortes Evitáveis, 2000/2001 e 2007/2008, Londrina (PR).

\begin{tabular}{|c|c|c|c|c|c|c|c|c|c|c|}
\hline \multirow[b]{3}{*}{ Grupo de causas } & \multicolumn{5}{|c|}{ Baixo peso ao nascer } & \multicolumn{5}{|c|}{ Peso adequado ao nascer } \\
\hline & \multicolumn{2}{|c|}{$\underline{2000 / 2001}$} & \multicolumn{2}{|c|}{$2007 / 2008$} & \multirow[b]{2}{*}{ VP } & \multicolumn{2}{|c|}{$2000 / 2001$} & \multicolumn{2}{|c|}{$2007 / 2008$} & \multirow[b]{2}{*}{ VP } \\
\hline & $\mathbf{n}$ & TMI & $\mathbf{n}$ & TMI & & $\mathbf{n}$ & TMI & $\mathbf{n}$ & TMI & \\
\hline Causas de morte evitáveis & 97 & 6,30 & 79 & 5,98 & $-5,1$ & 32 & 2,08 & 18 & 1,36 & $-34,6$ \\
\hline Reduzíveis por ações de imunoprevenção & 1 & 0,06 & - & - & - & - & - & - & - & - \\
\hline $\begin{array}{l}\text { Reduzíveis por adequada atenção à mulher na } \\
\text { gestação e parto e ao feto e ao recém-nascido }\end{array}$ & 89 & 5,78 & 75 & 5,68 & $-1,7$ & 16 & 1,04 & 11 & 0,83 & $-20,2$ \\
\hline atenção à mulher na gestação & 54 & 3,51 & 55 & 4,16 & $+18,5$ & 5 & 0,32 & 4 & 0,30 & $-6,3$ \\
\hline atenção à mulher no parto & 29 & 1,88 & 20 & 1,51 & $-19,7$ & 7 & 0,45 & 4 & 0,30 & $-33,3$ \\
\hline atenção ao feto e ao recém nascido & 6 & 0,39 & - & - & - & 4 & 0,26 & 3 & 0,23 & $-11,5$ \\
\hline $\begin{array}{l}\text { Reduzíveis por ações adequadas de diagnóstico e } \\
\text { tratamento }\end{array}$ & 1 & 0,06 & - & - & - & 5 & 0,32 & 1 & 0,08 & $-75,0$ \\
\hline $\begin{array}{l}\text { Reduzíveis por ações adequadas de promoção à saúde, } \\
\text { vinculadas a ações adequadas de atenção à saúde }\end{array}$ & 6 & 0,39 & 4 & 0,30 & $-23,1$ & 11 & 0,71 & 6 & 0,45 & $-36,6$ \\
\hline Causas de morte mal-definidas & 1 & 0,06 & - & - & - & 3 & 0,19 & 1 & 0,08 & $-57,9$ \\
\hline Demais causas (não claramente evitáveis) & 25 & 1,62 & 20 & 1,51 & $-6,8$ & 22 & 1,43 & 30 & 2,27 & $+58,7$ \\
\hline Total & 123 & 7,99 & 99 & 7,50 & $-6,1$ & 57 & 3,70 & 49 & 3,71 & $+0,3$ \\
\hline
\end{tabular}

n: número de óbitos; TMI: taxa de mortalidade infantil; VP: variação percentual das taxas. 
subgrupo, em particular pelo aumento de transtornos maternos hipertensivos (código P00.0) como causa básica de morte.

Entre os nascidos com peso adequado, a taxa de mortalidade infantil por causas evitáveis diminuiu 34,6\% (Tabela 2), com redução para todos os subgrupos. Chama atenção, nesse grupo de NV, o aumento relativo de $58,7 \%$ na taxa de mortalidade por causas não claramente evitáveis que, em 2007/2008, passou a ser maior que a taxa de mortalidade por causas evitáveis. Esse aumento foi acompanhado pela maior frequência de malformações congênitas como causa básica de morte nesses NV com peso adequado.

A análise de correspondência evidenciou, para os NV de 2000/2001, um perfil de morte representado principalmente por óbitos evitáveis por adequada atenção à mulher no parto e por adequada promoção/atenção à saúde. Em 2007/2008, as mortes evitáveis por adequada atenção à $m u$ lher na gestação e as não claramente evitáveis exerceram papel principal na definição desse perfil (Figura 1).

Os subgrupos de óbitos evitáveis por adequada atenção ao recém-nascido e por adequado diagnóstico e tratamento foram os que mais contribuíram para a formação da dimensão resultante da análise, com inércia relativa de, respectivamente, $22,5 \%$ e $24,0 \%$. Os biênios contribuíram de modo semelhante para a dimensão, com inércia relativa de $48,9 \%$ para $2000 / 2001$ e de $51,1 \%$ para 2007/2008.

Em 2000/2001, a análise de correspondência revelou que o perfil de mortes dos nascidos com baixo peso e o dos nascidos com peso adequado foram idênticos e estiveram mais próximos de causas evitáveis por adequada atenção à mulher no parto e por adequada promoção/atenção à saúde (Figura 2). Por outro lado, em 2007/2008, essa análise mostrou um perfil de mortalidade de nascidos com baixo peso mais próximo de causas evitáveis por adequada atenção à mulher na gestação (Figura 2), enquanto que o de nascidos com peso adequado esteve mais próximo das causas não claramente evitáveis (Figura 2).

Os óbitos evitáveis por adequada atenção ao recém-nascido foram os que mais contribuíram para a dimensão da análise de correspondência dos óbitos de nascidos com baixo peso, e os claramente não evitáveis por adequado diagnóstico e tratamento, para a dos óbitos de nascidos com peso adequado (Figura 2). Os biênios contribuíram de modo semelhante para a formação da dimensão nas duas análises.

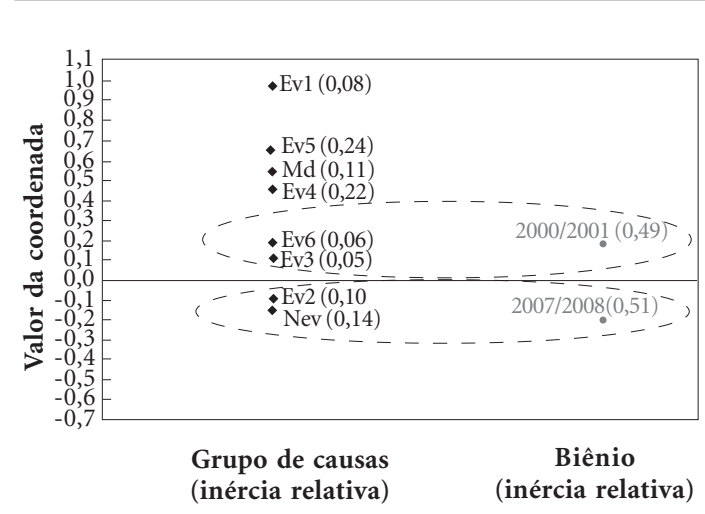

Legenda: Ev1 - óbitos evitáveis por ações de imunoprevenção; Ev2 óbitos evitáveis por adequada atenção à mulher na gestação; Ev3 óbitos evitáveis por adequada atenção à mulher no parto; Ev4 óbitos evitáveis por adequada atenção ao recém-nascido; Ev5 óbitos evitáveis por adequado diagnóstico e tratamento; Ev6 - óbitos evitáveis por adequada promoção/atenção à saúde; $\mathrm{Md}$ - Causas mal definidas; Nev - Causas não claramente evitáveis.

Figura 1. Comparação dos perfis de óbitos infantis de 2000/2001 e de 2007/2008 por análise de correspondência, Londrina (PR).

\section{Discussão}

Nos dois biênios estudados, a mortalidade infantil concentrou-se no período neonatal precoce e a maioria dos óbitos foi considerada evitável, especialmente por adequada atenção à mulher durante a gestação. De acordo com as taxas de mortalidade, as mortes evitáveis por adequada atenção à mulher na gestação foram mais frequentes entre nascidos com baixo peso e as não claramente evitáveis entre crianças com peso adequado, tanto em 2000/2001 como em 2007/2008. Entretanto, a análise de correspondência mostrou que esses dois subgrupos de causas estiveram mais próximos do perfil de morte identificado no biênio mais recente.

Aproximadamente sete em cada 10 óbitos infantis foram considerados evitáveis por intervenções do SUS, tanto no primeiro $(71,6 \%)$ como no segundo $(65,5 \%)$ período estudado, percentuais próximos aos encontrados em outros estudos brasileiros que utilizaram essa mesma classificação $\mathrm{o}^{4,8,14}$ ou que adotaram os critérios da Fundação SEADE ${ }^{15-18}$.

As mortes evitáveis por adequada atenção à mulher durante a gestação e o parto e ao feto e ao recém-nascido foram as mais frequentes. No entanto, observou-se comportamento diferenciado entre os subgrupos desse conjunto de causas: enquanto houve redução das mortes evitáveis por 

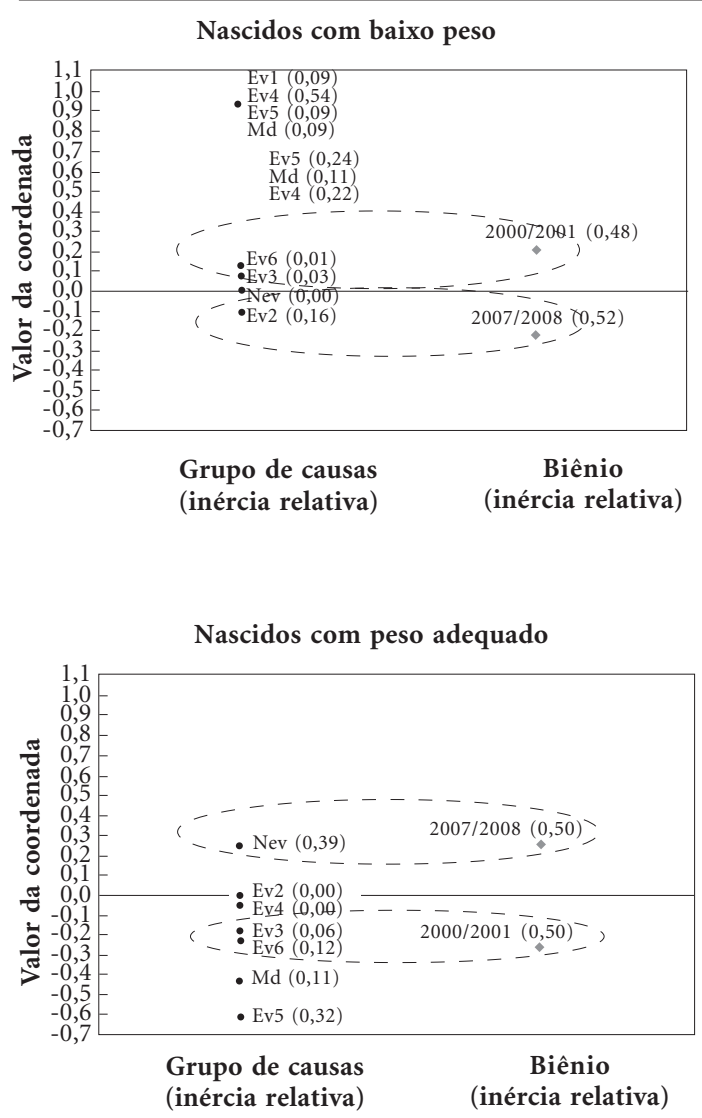

Legenda: Ev1 - óbitos evitáveis por ações de imunoprevenção; Ev2 - óbitos evitáveis por adequada atenção à mulher na gestação; Ev3 - óbitos evitáveis por adequada atenção à mulher no parto; Ev4 - óbitos evitáveis por adequada atenção ao recém-nascido; Ev5 - óbitos evitáveis por adequado diagnóstico e tratamento; Ev6 - óbitos evitáveis por adequada promoção/atenção à saúde; $\mathrm{Md}$ - Causas mal definidas; Nev Causas não claramente evitáveis.

Figura 2. Comparação do perfil dos óbitos infantis de 2000/2001 e de 2007/2008 resultantes de análise de correspondência, segundo categorias de peso ao nascer, Londrina (PR).

adequada atenção à mulher durante o parto e por adequada atenção ao feto e ao recém-nascido, as mortes evitáveis por adequada atenção à mulher durante a gestação apresentaram aumento. Gorgot et $a .^{8}$ também identificaram a atenção à mulher durante o pré-natal como principal responsável pelos óbitos evitáveis de menores de cinco anos em uma coorte de nascidos vivos de Pelotas (RS). Os autores atribuem esse achado a limitações na qualidade do pré-natal e ao aumento de nascimentos prematuros naquele município.

Em Londrina, entre os períodos estudados, houve importante expansão no número de equi- pes atuantes da ESF: de quatro para 93, em 2002, passando a abranger $70 \%$ da população urbana e $100 \%$ da rural, resultando em melhorias quantitativas e no acesso à atenção pré-natal. Todavia, avanços na qualidade desse serviço podem não ter acompanhado sua expansão, como assinalado por outros estudos brasileiros ${ }^{4,8}$. O aumento da frequência de prematuros no Município $^{7,15}$, assim como em outras localidades ${ }^{19}$, também pode ter contribuído para a predominância e o aumento das mortes evitáveis por adequada atenção à mulher durante a gestação, considerando que várias causas de prematuridade têm origem no período gestacional.

Outro aspecto a se considerar nesta análise é que houve melhora da qualidade da informação sobre as causas de morte no biênio mais recente, com redução das mal definidas e maior especificidade das demais, possivelmente devido ao aperfeiçoamento da atuação do CMPMMI. Isto pode ter contribuído para o aumento da frequência de óbitos evitáveis por adequada atenção à mulher na gestação e para a redução daquelas atribuídas à adequada atenção à mulher no parto.

Sabe-se que, na maioria das mortes neonatais, a causa inicial do processo que culminou no óbito, ou causa básica, está relacionada a morbidades maternas e a intercorrências durante a gravidez $^{20}$. Estas causas iniciais, porém, nem sempre são informadas na declaração de óbito, prejudicando a qualidade da informação e tornando o perfil de mortes mais próximo de causas terminais ${ }^{21} \mathrm{e}$, portanto, mais relacionadas ao parto do que à gestação. A melhora da informação sobre afecções que se iniciaram na gestação, como os transtornos maternos hipertensivos, e que resultaram em outros problemas e no óbito da criança após o nascimento, não pode ser descartada como hipótese para justificar parte do aumento das causas evitáveis por adequada atenção à mulher durante a gestação.

As mortes evitáveis por ações adequadas de diagnóstico e tratamento apresentaram a maior redução entre os biênios, porém os pequenos números recomendam cautela na análise. Essas mortes, em sua maioria, estão relacionadas a afecções que, em geral, não exigem tecnologias de custo elevado para seu diagnóstico e tratamento. Suas complicações, frequentemente, podem ser evitadas mediante acompanhamento da criança, buscando identificar situações de risco para que intervenções ocorram em tempo oportuno ${ }^{22}$.

Malta et al. ${ }^{4}$, em estudo sobre mortes infantis evitáveis no Brasil entre 1997 e 2006, também destacam a maior facilidade de acesso a interna- 
ções pelo SUS como medida que pode ter impactado de forma positiva na redução de mortes evitáveis por ações adequadas de diagnóstico e tratamento, sobretudo das afecções respiratórias, principais representantes desse grupo de causas no País e no presente estudo. Esses e outros autores também assinalam a possível contribuição da melhoria das condições de vida da população na redução de mortes infantis, em especial pela ampliação do acesso à educação e pela diminuição na desigualdade de renda ${ }^{4,23}$.

Quanto ao grupo das mortes evitáveis por ações de promoção à saúde, vinculadas a ações de atenção à saúde, as decorrentes de causas externas, em particular por aspiração de conteúdo gástrico, foram as de maior destaque. Estes eventos comumente ocorrem sem assistência médica, no local do acidente ou a caminho do serviço de urgência ${ }^{24}$. Tais características apontam para a importância de ações de prevenção em relação aos riscos associados a cada etapa do desenvolvimento da criança ${ }^{24}$ e o acesso rápido a orientações adequadas em caso de acidentes.

Paralelamente à observação de redução de mortes infantis por causas evitáveis, foi evidenciado aumento das mortes por causas não claramente evitáveis no biênio recente, com destaque para as malformações congênitas. Observou-se diminuição de malformações inespecíficas e aumento das do aparelho circulatório como causa básica de morte, o que confirma a melhora da especificidade da informação sobre a causa básica de morte também nos casos de malformações congênitas, medida importante tanto para o monitoramento dessas causas ${ }^{21}$ como para adequada atenção a crianças com esta condição pelos serviços de saúde.

As mortes decorrentes de malformações congênitas são consideradas de difícil redução, pois, em sua maioria, são de etiologia desconhecida. Entretanto, ações desenvolvidas no planejamento familiar e no pré-natal, como a suplementação vitamínica e com ácido fólico e o controle do diabetes materno podem colaborar na sua prevenção ${ }^{25}$. Além disso, em caso de detecção de malformação congênita durante o pré-natal, o encaminhamento da gestante para acompanhamento em serviços especializados e a programação do parto e do atendimento ao recém-nascido podem reduzir mortes infantis por essa causa ${ }^{26}$.

Estudos têm apontado a necessidade de aprofundar discussões sobre a melhor maneira de incorporar o peso ao nascer à classificação da evitabilidade de óbitos infantis s,6,27 $^{5}$ Por um lado, destaca-se a importância de se considerar, como evento-sentinela, óbitos ocorridos entre crianças com peso igual ou superior a $2500 \mathrm{~g}$, por sua relação com a qualidade do cuidado no pré-natal, no parto e na assistência ao recém-nascido ${ }^{27}$ e, por outro, a relevância do estabelecimento, ou não, de pontos de corte no peso ao nascer para posterior atribuição de evitabilidade aos óbitos infantis ${ }^{5}$, por sua relação com a viabilidade do recém-nascido.

Nesta pesquisa, optou-se por manter os óbitos de recém-nascidos com peso inferior a $1500 \mathrm{~g}$ na análise, primeiro pela semelhança de suas causas com as de óbitos de recém-nascidos com peso entre 1500 e $2499 \mathrm{~g}$ que, em sua maioria, correspondem a mortes evitáveis por adequada atenção à mulher na gestação. Segundo, porque o objetivo do estudo foi identificar o perfil de mortes infantis segundo os diferentes agrupamentos de causas evitáveis, incluindo aquelas que se originaram na gravidez e que resultaram em recémnascidos muito prematuros, e não verificar possíveis condições de sobrevivência após o nascimento vivo.

Embora os óbitos de nascidos vivos com baixo peso, em ambos os períodos, tenham ocorrido mais frequentemente por causas evitáveis por adequada atenção à mulher na gestação, a análise de correspondência revelou perfil de morte diferenciado para os biênios pesquisados. Em 2000/ 2001, as mortes evitáveis por adequada atenção à mulher no parto e por adequada promoção/atenção à saúde foram mais representativas da mortalidade evitável em crianças de baixo peso e, em 2007/2008, os óbitos evitáveis por adequada atenção à mulher na gestação ganharam destaque. É possível que este resultado esteja relacionado, como comentado anteriormente, à melhora na especificidade da causa básica de morte e ao aumento de nascimentos prematuros em anos recentes $^{7,15}$, resultando em mudanças no perfil de evitabilidade dos óbitos de nascidos vivos com baixo peso. Mesmo assim, estes achados reforçam a importância de qualificar os serviços disponíveis no SUS voltados ao atendimento prénatal ${ }^{4}$.

Entre os nascidos vivos com peso adequado, observou-se redução da taxa de mortalidade evitável por todos os subgrupos de causas. No biênio mais recente, as mortes não claramente evitáveis passaram a ser as principais representantes dos óbitos de crianças com peso adequado e, neste grupo de causas, as malformações congênitas do aparelho circulatório foram as mais frequentes. A mudança no perfil de mortalidade desses recém-nascidos sugere possível atuação positiva 
dos serviços de saúde na atenção e no acompanhamento da criança após o nascimento.

Vale ressaltar que a análise de correspondência é uma técnica exploratória de dados e foi utilizada, na presente pesquisa, para a classificação dos grupos de causas de mortes segundo os biênios selecionados a fim de visualizar o perfil de óbitos infantis para cada período e possíveis modificações. A técnica não permite estabelecer a significância estatística dos perfis identificados e nem avaliar o efeito independente de cada grupo de morte (categoria) na construção do perfil ${ }^{28}$. Entretanto, os perfis resultantes da análise de correspondência confirmam as hipóteses aventadas por análises das taxas e variações percentuais dos grupos de causas analisados. As mortes não claramente evitáveis ganharam importância no período mais recente, particularmente entre os nascidos com peso adequado. As mortes evitáveis por adequada atenção à mulher na gestação destacaram-se entre as causas consideradas evitáveis em ambos os períodos analisados e com incremento no biênio mais recente, particularmente entre nascidos com baixo peso, o que reforça a importância de medidas de prevenção e de diagnóstico e tratamento precoces de agravos durante a gestação, especialmente daquelas com potencial de prevenção do parto prematuro.

\section{Colaboradores}

HG dos Santos, SM de Andrade e AMR Silva participaram da concepção do projeto, tabulação e análise dos dados, da redação e da revisão crítica do artigo. TAF Mathias, LL Ferrari e AE Mesas participaram da análise dos dados, da redação e da revisão crítica do artigo.

\section{Agradecimentos}

Conselho Nacional de Desenvolvimento Científico e Tecnológico ( $\mathrm{CNPq})$, por bolsa de produtividade em pesquisa, Fundação Araucária, por bolsa técnico, e ao Ministério da Saúde/SCTIE/ Decit, CNPq e Fundação Araucária pelo auxílio financeiro. 


\section{Referências}

1. Barros FC, Matijasevich A, Requejo JH, Giugliani E, Maranhao AG, Monteiro CA, Barros AJ, Bustreo F, Merialdi M, Victora CG. Recent trends in maternal, newborn, and child health in Brazil: progress toward Millennium Development Goals 4 and 5. Am J Public Health. 2010; 100(10):1877-1889.

2. Victora CG, Aquino EM, Leal MC, Monteiro CA, Barros FC, Szwarcwald CL. Maternal and child health in Brazil: progress and challenges. Lancet 2011; 377(9780):1863-1876.

3. França E, Lansky S. Mortalidade infantil e neonatal no Brasil: situação, tendências e perspectivas. [página na Internet]. [acessado 2011 ago 21]. Disponível em: http://www.abep.nepo.unicamp.br/encontro2008/ docsPDF/ABEP2008_1956.pdf.

4. Malta DC, Duarte EC, Escalante JJ, Almeida MF, Sardinha LM, Macario EM, Monteiro RA, Morais Neto OL. Mortes evitáveis em menores de um ano, Brasil, 1997 a 2006: contribuições para a avaliação de desempenho do Sistema Único de Saúde. Cad Saude Publica 2010; 26(3):481-491.

5. Malta DC, Duarte EC, Almeida MF, Dias MAS, Morais Neto OL, Moura L, Ferraz W, Souza MFM. Lista de causas de mortes evitáveis por intervenções do Sistema Único de Saúde do Brasil. Epidemiol serv saúde 2007; 16(4):233-244.

6. Malta DC, Sardinha LMV, Moura L, Lansky S, Leal MC, Szwarcwald CL, França E, Almeida MF, Duarte EC. Atualização da lista de causas de mortes evitáveis por intervenções do Sistema Único de Saúde do Brasil. Epidemiol serv saúde 2010; 19(2):173-176.

7. Gabani FL, Sant'anna FHM, Andrade SM. Caracterização dos nascimentos vivos no município de Londrina (PR) a partir dos dados do SINASC. Ciênc cuid saúde 2010; 9(2):205-213.

8. Gorgot LRMR, Santos I, Valle N, Matisajevich A, Barros AJD, Albernaz E. Óbitos evitáveis até 48 meses de idade entre as crianças da Coorte de Nascimentos de Pelotas de 2004. Rev Saude Publica 2011; 45(2):334-342.

9. Graner VR, de Barros SM. Complicações maternas e ocorrências neonatais associadas às gestações múltiplas resultantes de técnicas de reprodução assistida. Rev Esc Enferm USP 2009; 43(1):103-109.

10. Departamento de Informática do Sistema Único de Saúde (Datasus). Informações de saúde. Nascidos Vivos - Paraná. [página na Internet]. [acessado 2010 ago 23]. Disponível em: http://tabnet.datasus.gov. $\mathrm{br} / \mathrm{cgi} /$ deftohtm.exe?sinasc/cnv/nvPR.def.

11. Machado CJ. A literature review of record linkage procedures focusing on infant health outcomes. Cad Saude Publica 2004; 20(2):362-371.

12. Carvalho MS, Struchiner CJ. Análise de correspondência: uma aplicação do método à avaliação de serviços de vacinação. Cad Saude Publica 1992; 8(3):287-301.

13. Brasil. Ministério da Saúde (MS). Conselho Nacional de Saúde. Resolução no 196 de 10 de outubro de 1996. Diretrizes e Normas Regulamentadoras de Pesquisas Envolvendo Seres Humanos. Diário Oficial da União 1996; 16 out.
14. Gastaud AL, Honer MR, Cunha RV. Mortalidade infantil e evitabilidade em Mato Grosso do Sul, Brasil, 2000 a 2002. Cad Saude Publica 2008; 24(7): 1631-1640.

15. Ferrari LS, Brito AS, Carvalho AB, Gonzales MR. Mortalidade neonatal no Município de Londrina, Paraná, Brasil, nos anos 1994, 1999 e 2002. Cad Saude Publica 2006; 22(5):1063-1071.

16. Monteiro RA, Schmitz BAS. Principais causas básicas da mortalidade infantil no Distrito Federal, Brasil: 1990 a 2000. Rev Bras Saude Mater Infant. 2004; 4(4):413-421.

17. Vidal SA, Frias PG, Barreto FMP, Vanderlei LCM, Felisberto E. Óbitos infantis evitáveis em hospital de referência estadual do Nordeste brasileiro. Rev Bras Saude Mater Infant. 2003; 3(3):281-289.

18. Ribeiro VS, Silva AA. Tendências da mortalidade neonatal em São Luís, Maranhão, Brasil, de 1979 a 1996. Cad Saude Publica 2000; 16(2):429-438.

19. Silveira MF, Santos IS, Matijasevich A, Malta DC, Duarte EC. Nascimentos pré-termo no Brasil entre 1994 e 2005 conforme o Sistema de Informações sobre Nascidos Vivos (SINASC). Cad Saude Publica 2009; 25(6):1267-1275.

20. Carvalho ML, Silver LD. Confiabilidade da declaração da causa básica de óbitos neonatais: implicações para o estudo da mortalidade prevenível. Rev Saude Publica 1995; 29(5):342-348.

21. Laurenti R, de Mello Jorge MH, Gotlieb SL. Mortalidade segundo causas: considerações sobre a fidedignidade dos dados. Rev Panam Salud Publica 2008; 23(5):349-356.

22. Caldeira AP, França E, Perpetuo IH, Goulart EM. Evolução da mortalidade infantil por causas evitáveis, Belo Horizonte, 1984-1998. Rev Saude Publica 2005; 39(1):67-74.

23. Garcia LP, Santana LR. Evolução das desigualdades socioeconômicas na mortalidade infantil no Brasil, 1993-2008. Cien Saude Colet 2011; 16(9):3717-3728.

24. Martins CB, Andrade SM. Acidentes com corpo estranho em menores de 15 anos: análise epidemiológica dos atendimentos em pronto-socorro, internações e óbito. Cad Saude Publica 2008; 24(9): 1983-1990.

25. Amorim MMR, Vilela PC, Santos ARVD, Lima ALMV, Melo EFP, Bernardes HF, Menezes Filho PFB, Guimarães VB. Impacto das malformações congênitas na mortalidade perinatal e neonatal em uma maternidade-escola do Recife. Rev Bras Saude Mater Infant 2006; 6(Supl. 1):s19-s25.

26. Gomes MRR, Costa JSD. Mortalidade infantil e as malformações congênitas no Município de Pelotas, Estado do Rio Grande do Sul, Brasil: estudo ecológico no período 1996-2008. Epidemiol serv saúde 2012; 21(1):119-128.

27. Lansky S, França E, Leal MC. Mortalidade perinatal e evitabilidade: revisão da literatura. Rev Saude Publica 2002; 36(6):759-772. 
28. Aranha RN, Faerstein E, Azevedo GM, Werneck G, Lopes CS. Análise de correspondência para avaliação do perfil de mulheres na pós-menopausa e o uso da terapia de reposição hormonal. Cad Saude Publica 2004; 20(1):100-108.

Artigo apresentado em 08/01/2013

Aprovado em 27/02/2013

Versão final apresentada em 18/03/2013 